\title{
Improving operational effectiveness of tactical master plans for emergency and elective patients under stochastic demand and capacitated resources
}

\author{
Ivo Adan ${ }^{1}$, Jos Bekkers ${ }^{2}$, Nico Dellaert ${ }^{3}$, Jully Jeunet ${ }^{4}$, Jan Vissers ${ }^{5}$ \\ ${ }^{1}$ Eindhoven University of Technology, Department of Mathematics and Computer Science, \\ Postbus 513, 5600MB Eindhoven, The Netherlands \\ iadan@,tue.nl \\ ${ }^{2}$ Erasmus University Medical Centre, Thorax Centre \\ Rotterdam, The Netherlands \\ j.a.bekkers@erasmusmc.nl \\ ${ }^{3}$ Eindhoven University of Technology, Industrial Engineering School \\ n.p.dellaert@tue.nl \\ ${ }^{4}$ CNRS, Lamsade, Université Paris Dauphine, Place de Lattre de Tassigny, \\ 75775 Paris Cedex 16, France \\ jully.jeunet@dauphine.fr \\ ${ }^{5}$ Erasmus University Medical Centre, institute for Health Policy and Management, Rotterdam NL \\ j.m.h.vissers@erasmusmc.nl
}

\begin{abstract}
This paper develops a two-stage planning procedure for master planning of elective and emergency patients while allocating at best the available hospital resources. Four types of resources are considered: operating theatre, beds in the medium and in the intensive care units, and nursing hours in the intensive care unit. A tactical plan is obtained by minimizing the deviations of the resources consumption to the target levels of resources utilization. Some capacity is reserved for emergency care. To deal with the deviation between actually arriving patients and the average number of patients on which the tactical plan is based, we consider the option of planning a higher number of patients (overplanning). To adapt the tactical plan to the actual stream of elective patients, we also consider flexibility rules. Overplanning and flexibility leads to a weekly schedule of elective patients. This schedule is modified to account for emergency patients. Scheduled elective patients may be cancelled and emergency patients may be sent to other hospitals. Cancellations rules for both types of patients rely on the possibility to exceed the available capacities. Several performance indicators are defined to assess patient service/dissatisfaction and hospital efficiency. Simulation results show a trade-off between hospital efficiency and patient service. We also obtain a rank of the different strategies: overplanning, flexibility and cancellation rules.
\end{abstract}

Keywords: operation theatre planning, intensive and medium care, emergency and elective patients, overplanning, cancellation rules, operational schedule of patients, master surgical schedule.

\section{Introduction}

Master surgical plans can contribute to a balanced use of resources such as beds, operating theatres and nursing staff. Beliën and Demeulemeester (2007) propose a three-stage procedure for developing effective operating room (OR) schedules with a multi-resource perspective: allocation of OR time to surgical specialties at strategic level, development of a master surgery schedule at tactical level and scheduling individual patients at operational level. Van Oostrum et al. (2008) build their two-phase decomposition approach around surgical procedure types: defining the mix of procedures to be performed and scheduling the surgery types. Both studies report on improved performance on the use of resources by using a cyclic master surgical plan.

However, the operational performance can differ strongly from the expected performance of the tactical plan for several reasons. One reason is that the tactical master plan needs to be translated into an operational plan used for scheduling the patients; the operational plan may deviate from the tactical plan due to short-term availability of resources or due to the need to 
schedule more patients because of an unacceptable rise in waiting time. Another reason can be emergency admissions, because they have priority to elective admissions. What happens when there are more emergencies arriving than expected? This may place more demand on intensive care (IC) beds. It may be necessary to cancel elective patients that will need an IC bed after the surgical procedure. Thus, the balanced use of resources of the master schedule may result in a much poorer performance on operational level. In this study we will consider the questions of how to develop more robust tactical plans and how to translate those plans into operational plans. First we will summarize the literature tackling this issue. Then we will introduce our approach to this problem.

Harper (2002) already emphasized the need for patient classification techniques for a better management of hospital resources. He develops a simulation model designed to assess the impact of the flow of emergency and elective patients over the use of three major resources: beds, operating theatres and medical staff. Simulation models are used for their predictive value as in the work of Ridge et al. (1998) which evaluates in particular the bed occupancy level when setting to some value the transfer rate of emergency patients to other hospitals. Emergency transferal as a way to manage patient overflow also is analysed in the paper of Litvak et al. (2008). The authors consider several regional hospitals that jointly reserve a number of beds in their intensive care unit for emergency patients. The authors develop an analytical approach to compute the number of regional beds to be reserved for any acceptance rate and show that cooperation leads to a higher acceptance rate with a lower number of reserved beds. One way to deal with overflow is to develop operational strategies to gain capacity. To that purpose, Cochran and Roche (2009) design a split patient flow model to treat lower acuity patients in a separate queue from higher acuity patients waiting for a bed.

On a more tactical level, the available capacity has to be divided over the elective patients and the emergencies. Dealing with emergency patients may amount to determine the level of capacity required to operate a pattern of elective patients so as to keep the deferral rate as low as possible. For instance, Utley et al. (2003) use generating functions to estimate the bed needs depending on overall levels of elective admissions and emergency arrivals. Lamiri et al. (2008) develop a stochastic model for operating room planning to deal with both an elective and emergency demand for surgery. The objective is to assign elective patients to periods so as to minimize the sum of elective patient related costs and overtime costs of operating rooms.

The purpose of this paper is two-fold. First we design an optimisation approach to determine a tactical plan with reserved capacity for emergency patients. Then we develop operational strategies to deal with the actual flow of elective and emergency patients on a weekly and daily basis. We will illustrate our approach with data related to thoracic surgery, using a setting from a Dutch Thorax Centre described hereafter. We consider several groups of patients according to their demand profile on resources; each of these groups being homogeneous in terms of the use of four main resources: operating theatres, beds in intensive care unit, beds in medium care unit and nursing staff. The groups can be linked to pathology categories or procedure types.

In the medium-run, operating theatre management requires a plan for all elective patients to be operated over a given horizon, generally four weeks, in order to allocate at best the major resources while not exceeding the available capacity. Part of this capacity is reserved for emergency patients. The problem is modelled as a mixed integer program the objective function 
of which consists in minimizing a weighted sum of deviations of resources consumptions to the target levels of resources utilization in order to obtain a smooth allocation of patients over the planning horizon. The resulting tactical plan is used to derive a weekly operational schedule for elective patients (see Figure 1). The actual operational performance will be influenced by actual lengths of stay and by emergency arrivals. In this planning phase, the only remaining control option is to cancel some operations. This final daily plan will be described as the execution plan.

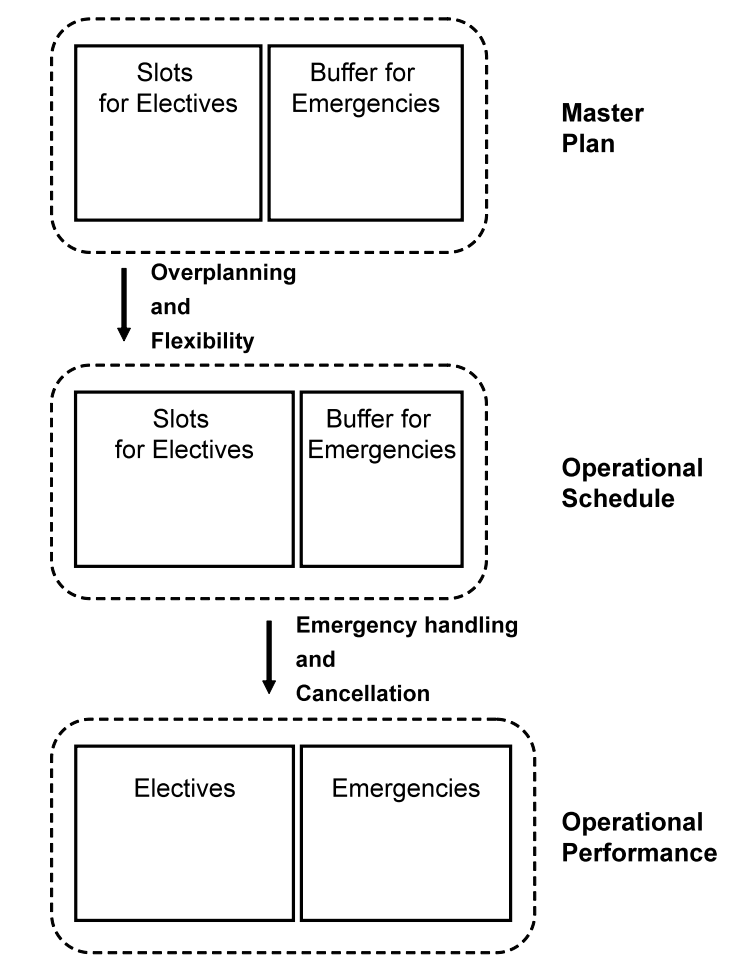

Figure 1. From master plan to operational performance

Since the actual flow of elective patients may differ from the average flow on the basis of which the tactical plan is determined, we use several strategies to plan the actual elective patients to obtain a better spread of resources usage over time. Two main strategies with different options are employed: overplanning and flexibility. Overplanning consists in computing the tactical plan with an increased number of elective patients to create a security slack. Flexibility leaves on each day the possibility of changing patient groups initially planned for operation. For instance, if no patients of a patient group are available on the waiting list, an elective patient of another group can be scheduled.

On a daily basis, we design several rules to deal with emergency patients. Some of them may be sent to other hospitals or elective patients may be postponed to avoid capacity excess. Handling emergency patients therefore leads to modify the operational plan of elective patients. The effectiveness of the flexibility strategies and the emergency scheduling rules is assessed by several indicators: the waiting time, operation cancellations and the number of schedule changes between the tactical plan and the operational plan. The performance will ultimately be expressed in terms of two indicators: patient service and hospital inefficiency. 
The central question we want to answer in this paper is which combinations of actions on the three planning levels lead to the most efficient performance and what recommendations can be given to make the final plan.

The remainder of the paper is organized as follows. Section 2 provides a general description of the problem and the approach we follow. In Section 3, we present the mathematical model used to obtain the planning of patients on a tactical level. Section 4 describes the flexible scheduling rules designed to obtain an operational schedule on a weekly basis. We then turn to a presentation of a daily scheduling algorithm we developed to deal with emergency patients. We finally present several indicators we defined to assess the performance of the developed strategies and algorithm. Numerical experiments are discussed in Section 5. Section 6 draws some conclusions and formulates recommendations for further work.

\section{Problem general description}

In any hospital, the patient flow consists of planned elective patients and emergency patients requiring immediate surgery. On a tactical level, elective patients are planned so as to obtain an efficient use of the resources over a certain planning horizon (usually four weeks). Part of the maximum capacity of each resource is reserved for emergency patients. The resulting tactical plan is used as a guideline with various degrees of flexibility to decide upon the operational scheduling of elective and emergency patients on a weekly and daily basis.

Following previous research in the hospital planning area for cardiothoracic surgery (Vissers et al., 2005; Adan et al., 2009), we consider several categories of patients, each of these categories being homogeneous in terms of resources consumption. Some categories of patients are admitted to the Medium Care unit (MC) one day before operation unless they are emergency patients. Patients are then transferred to the operating theatre (OT) with an operation duration that depends on the pathology category. After the operation, they stay for some days in an Intensive Care (IC) unit where they benefit from specialized nursing care. After recovery they may stay in the MC unit for a few days. Then patients are discharged to return home. We consider four major resources: operating theatre hours (OT), Intensive Care beds (IC), Intensive Care nursing hours $(\mathrm{NH})$ and Medium Care beds (MC). Each of these resources is available in limited quantity on each day, with a maximum capacity on weekdays that may differ from the week-end capacity. Patient categories are characterized by several data. The operation duration is assumed to be deterministic as in this paper we will not focus on overrun of OT sessions. The number of preoperative days spent in the MC unit is also deterministic. The length of stay in either IC unit or MC unit is stochastic, the distribution of which is estimated from empirical observations. The use of distributions instead of averages improves the quality of the master plan (Adan et al., 2009). Each patient group needs a certain number of nursing hours in the intensive care unit; the number of hours needed on a certain day depends on the number of post-operative days already spent by the patient in the IC unit (usually patients require more IC nursing care on the first and second day after operation than in any other day).

In the medium-run, the OT management faces the problem of determining a tactical plan for all elective patients so as to allocate at best the major resources while operating all patients that are expected during a "typical" horizon. In the next section, we formulate this problem as a mixed integer program. The objective is to minimize the weighted sum of deviations between the consumption of resources and target levels of resources utilization. The constraints of the plan 
ensure that all elective patients of each category are operated within the planning horizon and that expected resources consumptions exceed as little as possible the available capacities. The resulting solution to this planning problem consists of daily planned operations for each category. This tactical plan is then used in the short run to schedule elective patients on a weekly basis, using overplanning or flexibility strategies to handle deviations between actual flow of patients and the average flow used to derive the tactical plan. The daily flow of emergency patients is divided into day arrivals and night arrivals. Night emergency patients are all operated during the night shift or during the subsequent day shift. During the day, the projected use of resources by emergency and elective patients is computed and compared to the available capacity. To avoid capacity excess, we define some rules to postpone elective patients and to send some emergency patients to other hospitals. These operational strategies are described in Section 4.

\section{Mathematical model for the tactical planning with emergency patients}

In this section we formulate the tactical planning problem as a mixed integer linear program. We adopt the notations and definitions in Table 1.

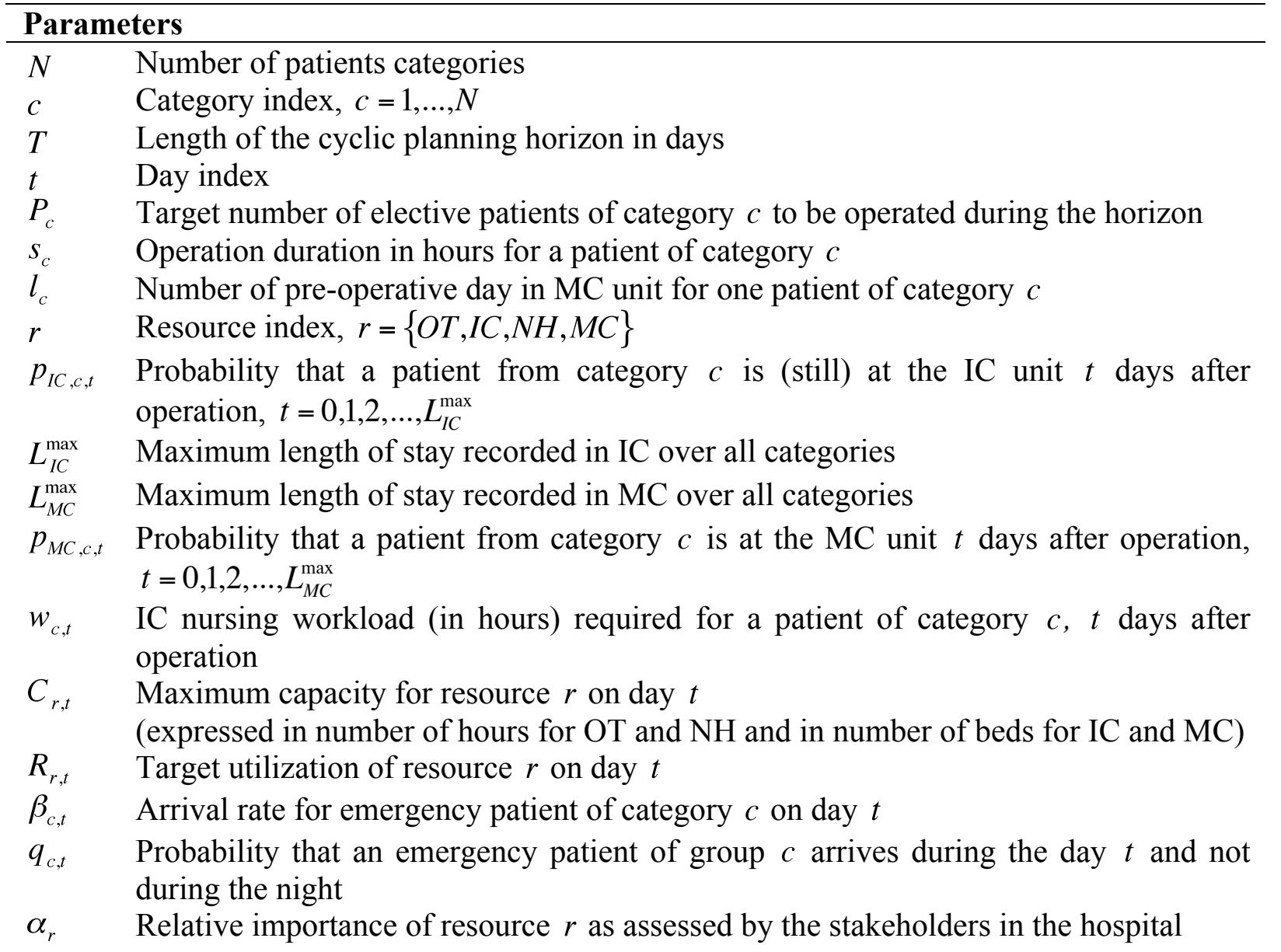




\section{Variables}

$X_{c, t} \quad$ Number of patients from category $c$ operated on day $t$, with $c=1, \ldots, N$ and $t=1, \ldots, T$

$O_{r, t} \quad$ Over utilization of resources relative to the target use for resource $r$ on day $t$, $r=\{O T, I C, N H, M C\}$ and $t=1, \ldots, T$

$U_{r, t} \quad$ Under utilization of resources relative to the target use for resource $r$ on day $t$, $r=\{O T, I C, N H, M C\}$ and $t=1, \ldots, T$

$E_{r, t} \quad$ Overuse of resource $r$ on day $t$ compared to the maximum capacity

\section{Table 1. Notations}

Note that the target utilization can be used to reserve capacity for emergercies. The objective is to determine the variables $X_{c, t}$ satisfying certain constraints and for which the daily expected utilization of each resource deviates as least as possible from the daily target consumption. Planning of patients $\left\{X_{c, t}\right\}$ must also minimize the overuse of resources relative to the maximum capacities. Thus, the objective function to be minimized can be written as

$$
\sum_{r=\{O T, I C, N H, M C\}} \alpha_{r} \sum_{t=1}^{T}\left(O_{r, t}+U_{r, t}+b \cdot E_{r, t}\right)
$$

where $b \geq 0$ is a constant penalizing capacity excess.

The total number of patients of group $c$ to be operated over the $T$-day cycle should be equal to the target patient throughput $P_{c}$. Hence

$$
\sum_{t=1}^{T} X_{c, t}=P_{c}, \quad c=1, \ldots, N
$$

The expected utilization of the OT by both elective and emergency patients must satisfy

$$
\left.\begin{array}{rl}
\sum_{c=1}^{N} s_{c} X_{c, t}+\sum_{c=1}^{N} s_{c} q_{c, t} \beta_{c, t} & \leq C_{O T, t}+E_{O T, t} \\
& \leq R_{O T, t}+O_{O T, t} \\
& \geq R_{O T, t}-U_{O T, t}
\end{array}\right\} \quad t=1, \ldots, T
$$

The expected number of beds in IC must also satisfy the three following inequalities

$$
\left.\begin{array}{rl}
\sum_{c=1}^{N} \sum_{j=0}^{L_{I C}^{\max }} p_{I C, c, j} X_{c, t-j}+\sum_{c=1}^{N} \sum_{j=0}^{L_{I C}^{\max }} p_{I C, c, j} \beta_{c, t-j} & \leq C_{I C, t}+E_{I C, t} \\
& \leq R_{I C, t}+O_{I C, t} \\
& \geq R_{I C, t}-U_{I C, t}
\end{array}\right\} \quad t=1, \ldots, T
$$


In the above constraints we used the convention that the subscript $t-j$ in $X_{c, t-j}$ should be treated modulo $T$ : day 0 is the same as day $T$, day -1 is the same as day $T-1$ and so on. For the expected number of nursing hours in IC, we must have

$$
\left.\begin{array}{rl}
\sum_{c=1}^{N} \sum_{j=0}^{L_{I C}^{\max }} w_{c, j} p_{I C, c, j} X_{c, t-j}+\sum_{c=1}^{N} \sum_{j=0}^{L_{I C}^{\max }} w_{c, j} p_{I C, c, j} \beta_{c, t-j} & \leq C_{N H, t}+E_{N H, t} \\
& \leq R_{N H, t}+O_{N H, t} \\
& \geq R_{N H, t}-U_{N H, t}
\end{array}\right\} \quad t=1, \ldots, T
$$

Similarly, the expected number of beds in MC must satisfy

$$
\left.\begin{array}{rl}
\sum_{c=1}^{N} \sum_{j=1}^{l_{c}} X_{c, t+j}+\sum_{c=1}^{N} \sum_{j=0}^{L_{M C}^{\max }} p_{M C, c, j} X_{c, t-j}+\sum_{c=1}^{N} \sum_{j=0}^{L_{M C}^{\max }} p_{M C, c, j} \beta_{c, t-j} & \leq C_{M C, t}+E_{M C, t} \\
& \leq R_{M C, t}+O_{M C, t} \\
& \geq R_{M C, t}-U_{M C, t}
\end{array}\right\} \quad t=1, \ldots ., T
$$

As operating rooms on weekends are dedicated only to emergency patients, we have to require that

$$
X_{c, t}=0 \text { and } X_{c, t+1}=0 \quad t=6+7 \cdot(j-1) ; \quad j=1, \ldots,(T / 7) ; \quad c=1, \ldots, N
$$

Our planning problem therefore consists in minimizing the objective function in (1) subject to constraints (2) to (7) and the integrality constraints (8):

$$
X_{c, t} \in\{0,1,2, \ldots\}, \quad c=1, \ldots, N ; t=1, \ldots, T
$$

The resources reserved by the tactical plan for the elective patients are based on the expected demand over the planning horizon. However if the average number of arriving elective patients is equal to the maximum number that can be treated, the system will become instable in the end. Thus, to obtain a feasible operational plan for elective patients, we will consider two options to create a security slack: overplanning and flexibility in patient groups. This will be explained in the next section.

\section{The operational plan}

The overplanning strategy increases the target throughput of patients $\left\{P_{c}\right\}_{c=1, \ldots, N}$ to create more opportunities for admitting patients. The flexibility strategy allows for the replacement of patients from one category by patients from another category to avoid unused capacity in some days. These strategies are combined to determine a short-term plan of elective patients one week in advance as patients are usually given a notice of admission one week in advance. We first 
describe the method to obtain such a plan in the absence of emergency patients and then present the algorithm to deal with emergency patients.

\subsection{Scheduling rules to obtain an operation plan of elective patients}

The scheduling rules used to build the operational plan of elective patients are a combination of different options for overplanning and patient group flexibility. For the sake of illustration, let us consider 3 categories of patient over a cycle of 7 days. Table 2 provides an optimal tactical plan corresponding to that problem.

\begin{tabular}{|c|c|c|c|c|c|c|c|c|c|}
\hline \multirow[b]{2}{*}{ Patient group } & \multicolumn{7}{|c|}{ Planned operation over a 7-day cycle } & \multirow{2}{*}{$\begin{array}{c}\text { Planned \# } \\
\text { patients }\left(P_{c}\right)\end{array}$} & \multirow{2}{*}{$\begin{array}{c}\text { Average } \neq \\
\text { patients }\end{array}$} \\
\hline & 1 & 2 & 3 & 4 & 5 & 6 & 7 & & \\
\hline Group 1 & 5 & 4 & 0 & 4 & 0 & 0 & 7 & 20 & 19.85 \\
\hline Group 2 & 2 & 1 & 2 & 1 & 0 & 0 & 0 & 6 & 5.72 \\
\hline Group 3 & 0 & 1 & 3 & 0 & 0 & 0 & 0 & 4 & 3.64 \\
\hline
\end{tabular}

Table 2. Example of an optimal tactical plan

Overplanning. The 'no-overplanning' option (O1) simply consists in calculating the tactical plan on the basis of the initial values of target: $\left\{P_{1}, \ldots, P_{N}\right\}$, by rounding up expected numbers of patients per category. We also consider two other options: the 'large overplanning' option (O2), which creates about 10 percent more admission opportunities and the 'intermediate overplanning' option (O3) about 5 percent. Table B6 in appendix B contains the exact target numbers.

Flexibility. Three flexibility options are considered to decide upon the patients that will be scheduled for operation, resulting from a comparison between the tactical plan and the actual elective patients in the queue. The different options result in an operational plan that will stick more or less closely to the tactical plan.

(F1) No Flexibility. In the 'no flexibility' option the reserved capacity is not used if there are no waiting patients of the planned category.

(F2) Large Flexibility. This option allows unplanned categories to be scheduled. Amongst the categories that are planned, we replace those with no waiting patient with other planned category for which there are waiting patients. The selected category for replacement is the one for which the product of the number of planned patients and the number of waiting patients is maximum. If such a category does not exist, we take an unplanned category with the longest waiting time. (F3) Intermediate flexibility. This option is identical to the previous one, but in case a planned category for replacement does not exist, we do not take any unplanned category. This results in no scheduling some waiting patients.

To illustrate the flexibility options, let us consider the planned number in the tactical plan on day 1: $\{5,2,0\}$ and assume the actual available patients are equal to $\{6,0,2\}$. In the 'no flexibility' option (F1) we will only schedule 5 patients of category 1 , as we do not allow for extension or replacement of patients. The 'large flexibility' option (F2) replaces the first patient of category 2 with the remaining patient of category 1 and the second patient of the second category with a third category patient, even if this category is not in the tactical plan. Thus (F2) leads to the schedule of patients: $\{6,0,1\}$. 
Under the 'intermediate flexibility' option (F3), the second category for which there is no actual patient is replaced with the first category, but the second patient of category 2 cannot be replaced with the third group because it is not in the tactical plan. Thus, (F3) leads to the following schedule of patients: $\{6,0,0\}$.

Combining the 3 options for overplanning and the 3 options for flexibility leads to 9 different flexible scheduling rules to obtain a short-run schedule of elective patients.

\subsection{Decision rules to deal with emergency patients}

By convention, we assume that a full working day starts in the evening with the night shift (from $6 \mathrm{pm}$ to $9 \mathrm{am}$ ). During this night shift, the patient flow consists of the remaining day emergencies (patients arrived in the previous day shift) and the night emergency arrivals. In the day shift, the flow is composed of the remaining night emergencies, the day emergency arrivals as well as the planned elective patients from the operational plan determined according to the method described in the previous subsection. Figure 2 illustrates the patient flow on any day $t$.

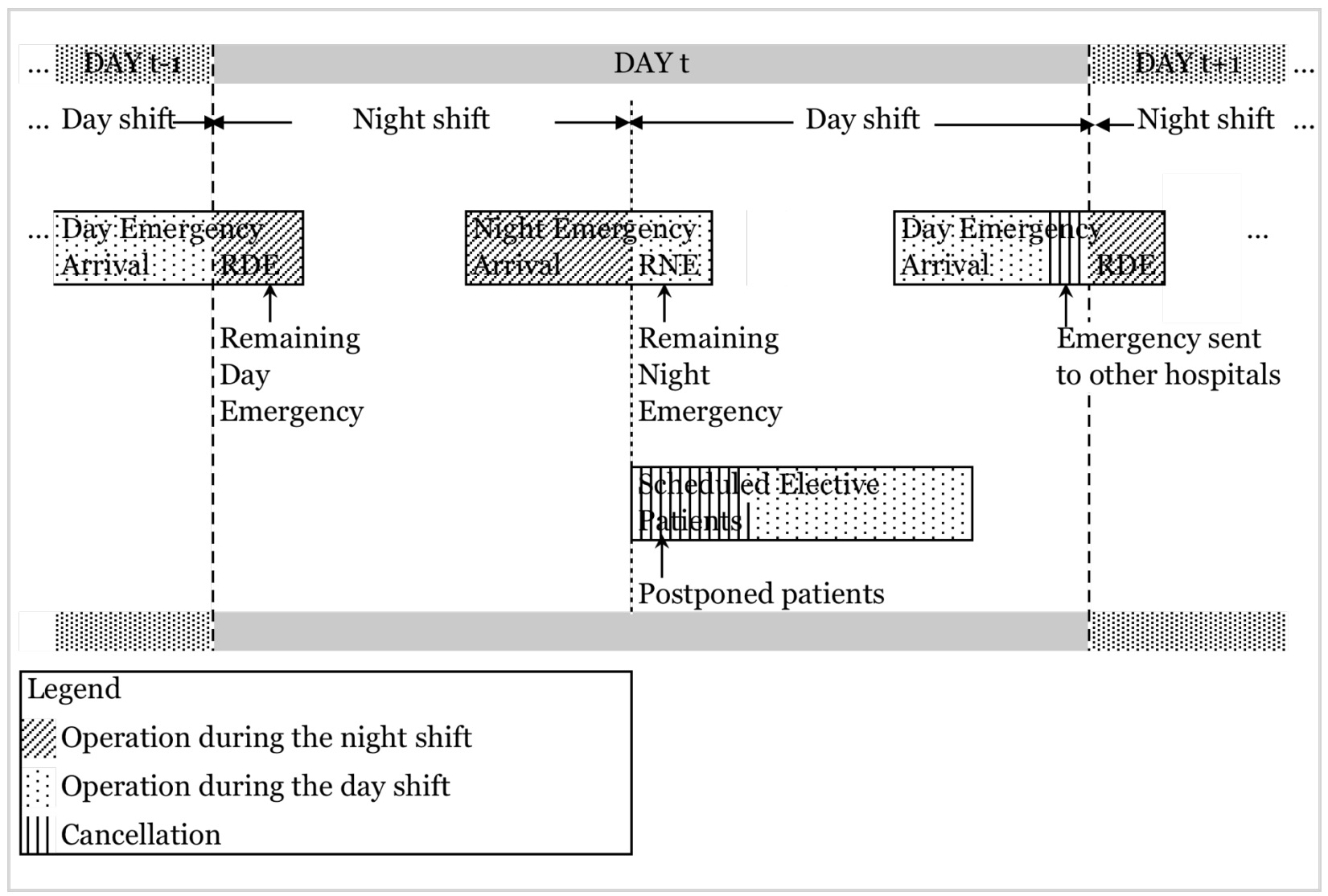

Figure 2. Patient flow on day $t$

As illustrated in this Figure, we first operate the emergency patients arrived during the day shift of the previous day. All night emergency arrivals are operated during the night shift so far as we can; the remaining night emergency patients are operated during the following day shift. At the beginning of each day shift, the projected consumption of resources is compared to the 
capacity which may lead to a revision of the planned elective patients to be operated. Some day emergency patients may also be sent to other hospitals to avoid a capacity violation

Figure 3 presents a simplified version of the daily scheduling algorithm for the elective patients and the emergencies; the detailed version can be found in Appendix A. The consumption of resource $r$ on day $t$ is denoted by $Q_{r, t}$. In the initialization step, we start with an empty system. We then fill the waiting list with arriving elective patients for 7 days to come. We determine an operational plan over the next 7 days, following one of the nine flexible scheduling rules described in section 4.1. Patients that have been scheduled are removed from the waiting list.

The night shift begins with the completion of the operation of the day emergency patients arrived during the previous day shift. All night emergencies are then operated in order of their arrival. We assume no night emergency patient is sent to another hospital because during the night, it is far more complicated to find a bed in another hospital than during the day shift.

The day shift begins with an estimation of the number of emergency patients that are expected during the day. We then compute the projected use of each resource over a horizon of $S$ days by (i) the already operated patients, (ii) the remaining night emergencies and (iii) the estimated day emergencies. The lengths of stay of the hospitalized patients are now assumed to be known exactly, whereas for the estimated day emergencies we still utilize the projected capacity use from the tactical plan. We consider all elective patients scheduled for operation on the current day, starting with the least crowded category and ending with the most crowded one. We apply to each of these patients the decision rule for cancellation (right side of Figure 3 ). The resources consumption of each additional patient is computed and added to the projected consumptions. If, for all resources and all periods from $t$ to $t+S$, the projected consumptions do not exceed the capacities multiplied by a coefficient $\rho(s)$, then the selected patient is operated. Coefficient $\rho(s)$ can be chosen as decreasing in time to reflect the fact that cancellations in a near future are less desirable than cancellations in a more distant future. The higher $\rho(s)$, the lower the risk of cancellation. For values of $\rho(s)$ above 1, the higher $\rho(s)$, the higher the risk of capacity violation. If, for one of the 4 resources in one of the period $t+s$, the consumption exceeds the capacity (multiplied by $\rho(s)$ ) then the operation is cancelled. The selected patient is put on the top of the waiting list and has to be scheduled again. Once all scheduled elective patients have been treated this way, we obtain a revised number of elective patients to be operated; these operations can no longer be cancelled.

Before the actual emergency patients are considered, the consumptions are actualized: we remove the estimated consumption by the expected day emergency patients. The day emergency patients are treated according to their arrival time: the earliest arrived patients are considered first. The cancellation rule is then applied to any patient in the same way as we did for scheduled elective patients. If the capacity (multiplied by $\rho(s)$ ) is exceeded, the patient is obviously not postponed but sent to another hospital. Once all day emergencies have been treated, the waiting list of elective patients on day $t+7$ is considered for planning. The waiting list is composed of actual arriving elective patients, postponed scheduled elective patients and patients that have not been scheduled for operation yet because of the chosen flexibility rule. 


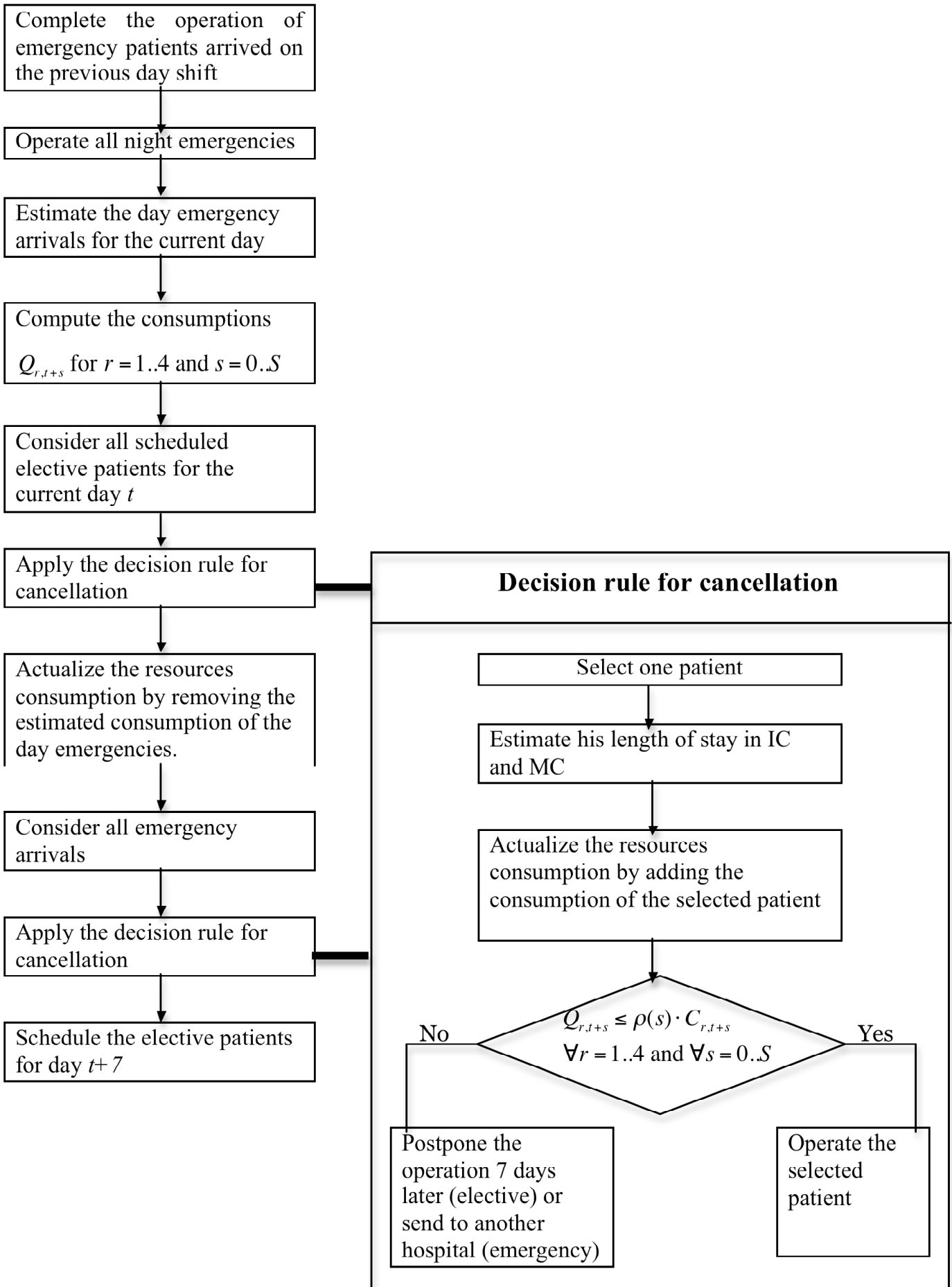

Figure 3. Simplified version of the daily scheduling algorithm

\subsection{Performance criteria}

The performance of the whole scheduling procedure (flexible scheduling rules and daily scheduling algorithm) will be assessed by several indicators reflecting the changes between the tactical plan, the operational schedule of elective patients and the stream of actually operated patients. 


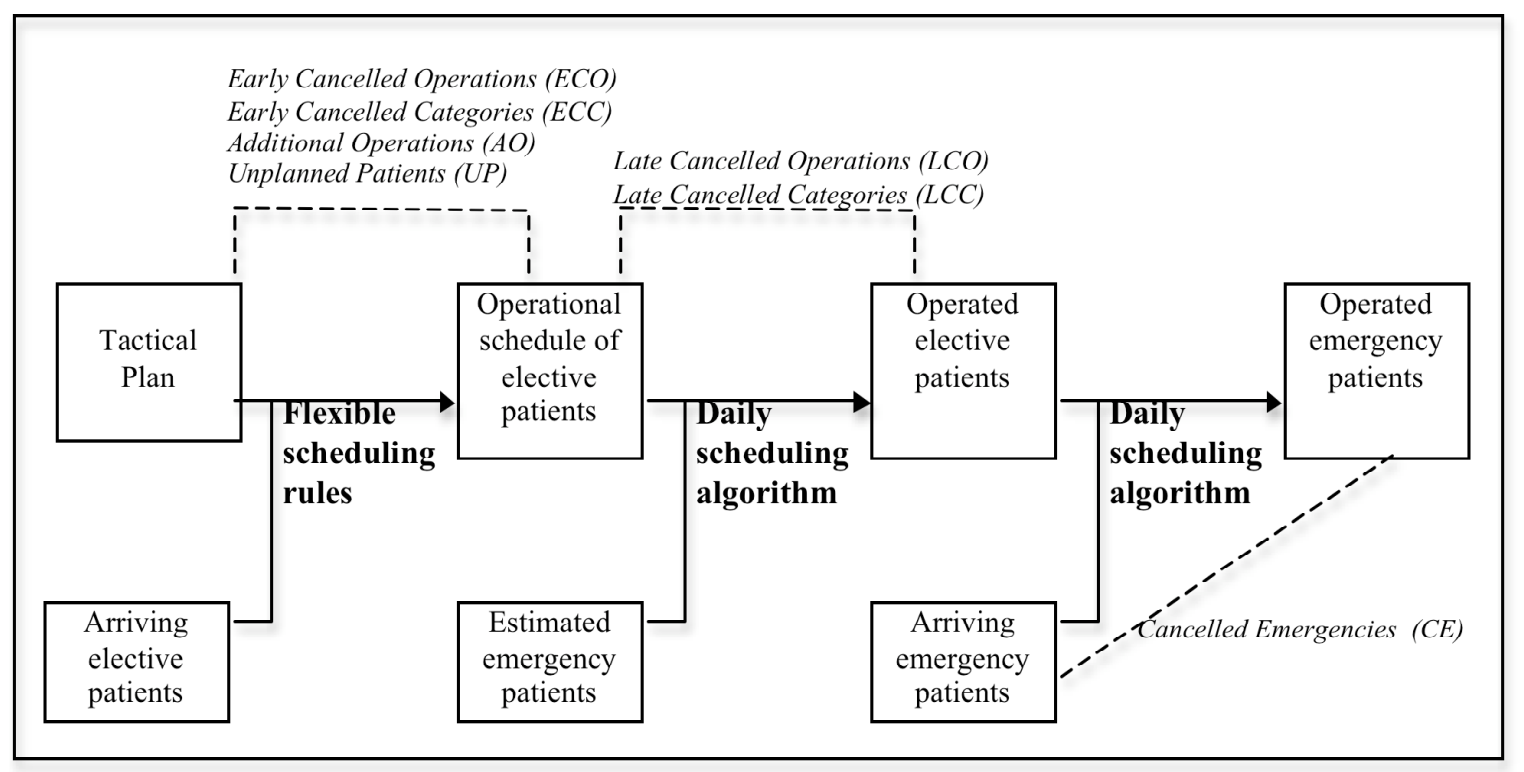

Figure 4. Plan changes in the 2-stage scheduling procedure

Figure 4 illustrates these plan changes in our 2-stage scheduling procedure. Using one of the nine flexible scheduling rules (Section 4.1) to deal with arriving elective patients leads to an operational plan that can differ from the initial tactical plan. Some categories of patients may be added or cancelled compared to the tactical plan. There can also be more or less operations. Then the cancellation rule is applied to the operational plan containing the scheduled elective patients. We refer to late cancellations, as these cancellations are decided on the day of operation.

Recall that $X_{c, t}$ is the variable designating the number of planned patients of category $c$ to be operated in period $t$ in the tactical plan. Further, let $S E P_{t}$ be the set of scheduled elective patients as determined in the operational plan. We have $S E P_{t}=\left\{a_{1, t}^{S E P}, \ldots, a_{c, t}^{S E P}, \ldots, a_{N, t}^{S E P}\right\}$, where $a_{c, t}^{S E P}$ is the number of elective patients of category $c$ scheduled for operation on day $t$. Let $A O P_{t}=\left\{a_{1, t}^{A O P}, \ldots, a_{c, t}^{A O P}, \ldots, a_{N, t}^{A O P}\right\}$ be the set of elective patients that have actually been operated (Already Operated Patients). The changes in the three plans (tactical plan, operational plan, executed plan) will be part of the hospital efficiency performance.

Table 3 provides the formal description of the single indicators that are combined to define compound indicators of patient service and hospital efficiency. Some indicators are weighted. We use the notation $\gamma$ to designate a weight. The value of the weights has been determined in cooperation with the hospital staff, reflecting financial and administrative consequences. For all indicators we take the average per 4-weeks cycle. The number of cycles included in a simulation is denoted by $\eta$; the total number of periods in a simulation thus equals $\eta T$. Variable $1_{\{\text {Cond 1;Cond } 2\}}$ takes a value of 1 if conditions 'Cond1' and 'Cond2' are true and zero otherwise. 
Single indicator

Compound indices of inefficiency
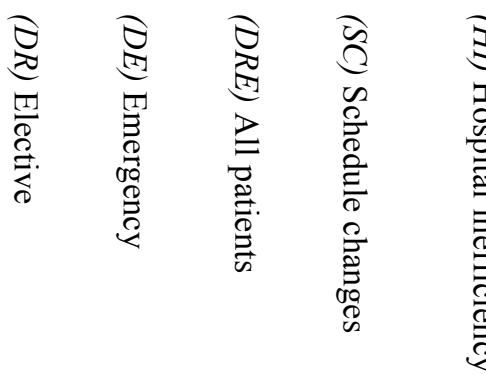

Exceeding

$$
E=\frac{1}{\eta} \sum_{r=1}^{4} \gamma_{E, r} \sum_{t=1}^{\eta T} \max \left(0, Q_{r, t}-C_{r, t}\right)
$$

100

\begin{tabular}{|c|c|c|c|c|c|c|}
\hline $\begin{array}{l}\text { Additional } \\
\text { Operations }\end{array}$ & $A O=\frac{1}{\eta} \sum_{t=1 c=1}^{\eta T} \sum_{c=1}^{N} \max \left(0, a_{c, t}^{S E P}-X_{c, t}\right) \cdot 1_{\left\{X_{c, t}>0\right\}}$ & & & & 5 & $5 \times 50$ \\
\hline $\begin{array}{l}\text { Unplanned } \\
\text { Patients }\end{array}$ & $U P=\frac{1}{\eta} \sum_{t=1}^{\eta T} \sum_{c=1}^{N} a_{c, t}^{S E P} \cdot 1_{\left\{X_{c, t}=0\right\}}$ & & & & 10 & $10 \times 50$ \\
\hline $\begin{array}{l}\text { Early } \\
\text { Cancelled } \\
\text { Operations }\end{array}$ & $E C O=\frac{1}{\eta} \sum_{t=1}^{\eta T} \sum_{c=1}^{N} \max \left(0, X_{c, t}-a_{c, t}^{S E P}\right) 1_{\left\{a_{c, t}^{S E P}>0\right\}}$ & & & & 1 & $1 \times 50$ \\
\hline $\begin{array}{l}\text { Early } \\
\text { Cancelled } \\
\text { Categories }\end{array}$ & $E C C=\frac{1}{\eta} \sum_{t=1}^{\eta T} \sum_{c=1}^{N} 1_{\left\{X_{c, t}>0 ; a_{c, t}^{S E P}=0\right\}}$ & & & & 2 & $2 \times 50$ \\
\hline $\begin{array}{l}\text { Late } \\
\text { Cancelled } \\
\text { Categories } \\
\end{array}$ & $L C C=\frac{1}{\eta} \sum_{t=1}^{T} \sum_{c=1}^{N} 1_{\left\{a_{c, t}^{S E P}>0 ; a_{c, t}^{A O P}=0\right\}}$ & & & & 3 & $3 \times 50$ \\
\hline $\begin{array}{l}\text { Weighted } \\
\text { Late } \\
\text { Cancelled } \\
\text { Operations }\end{array}$ & $\begin{array}{l}W L C O=\frac{1}{\eta} \sum_{k=1}^{7} \gamma_{W L C O, k} \cdot L C O_{k} ; \text { with } \\
L C O_{k}=\sum_{\substack{t \in\{1 . \eta T\} \\
t=k(\bmod 7)}} \sum_{c=1}^{N}\left(a_{c, t}^{S E P}-a_{c, t}^{A O P}\right)\end{array}$ & 1 & & 1 & & \\
\hline $\begin{array}{l}\text { Waiting } \\
\text { Time }\end{array}$ & $W T$ & 100 & & 100 & & \\
\hline $\begin{array}{l}\text { Cancelled } \\
\text { Emergencies }\end{array}$ & $C E$ & & 5 & 5 & & \\
\hline
\end{tabular}

Table 3. Single indicators and compound indices of inefficiency

Indicator of capacity excess $(E)$ is the weighed average of each resource capacity excess over a cycle, with $\gamma_{E, O T}=10, \gamma_{E, I C}=25, \gamma_{E, N H}=10, \gamma_{E, M C}=2$. The indicator of hospital inefficiency includes 100 times the value of $(E)$, as indicated in column (HI) of Table 3. Indicator $(A O)$ is the average number per cycle of additional scheduled patients in categories that were already planned in the tactical plan. Conversely, indicator $(U P)$ is the average number of shcheduled patients from categories that were not planned in the tactical plan. Early cancelled operations are represented by (ECO) which is the average number per cyle of planned operations that are finally not scheduled. Similarly, $(E C C)$ is the average number of early cancelled categories per cycle: 
categories that were in the tactical plan, but not in the operational plan. Late cancelled categories (LCC) express the average number per cycle of categories that were in the operational plan, but for which there is no actual operation. Indicator (WLCO) is the weighted sum for each day of the week of the percentage of scheduled elective patients that are finally not operated. Weights $\gamma_{W L C O, k}$ decrease with $k$ as cancelling the day of the operation is worse than cancelling an operation which was supposed to take place 6 days later. We thus chose $\left\{\gamma_{W L C O, k}\right\}_{k=1, \ldots, 7}=\{3,1,1,1,1,1,0\}$. The average waiting time $(W T)$ is the weighted average waiting time per category, with weights equal to the rate of (scheduled) patients in each category over all scheduled elective patients. The average waiting time $(W T)$ is expressed in number of days per cycle. It thus represents the waiting time between the day a patient arrives and the day in which the operation is scheduled. Indicator $(C E)$ is the average percentage per cycle of the number of emergency patients that are sent to other hospitals per cycle.

For patients, coumpound indices of inefficiency involve $(D R)$ : the dissatisfaction for regular (i.e. elective) patients, with $(D R)=(W L C O)+100 \cdot(W T)$; $(D E)$ : the dissatisfaction for emergency patients, with $(D E)=5 \cdot(C E)$; and $(D R E)$ : the dissatisfaction for both regular and emergency patients, with $(D R E)=(D E)+(D R)$. Index $(S C)$, reflecting schedule changes, is defined as $(S C)=5 \cdot(A O)+10 \cdot(U P)+1 \cdot(E C O)+2 \cdot(E C C)+3 \cdot(L C C)$. The hospital inefficiency index $(H I)$ includes both the capacity excess and the schedule changes, with $(H I)=100 \cdot(E)+50 \cdot(S C)$.

\section{Simulation}

\subsection{Data and parameter setting}

The data are partly based on the Thorax Center Rotterdam. A full presentation of the data is available in Appendix B. For each of the 3 overplanning options we solve the tactical planning problem. The corresponding mixed integer programs were solved using Cplex9.0 to obtain 3 tactical plans with an optimality gap ranging from 3 to $8 \%$, after a fixed computation time of 10 minutes. To obtain an operational plan, we apply the 3 flexibility options to the 3 tactical plans and thus obtain 9 different operational plans. The values we chose for parameter $\rho$ are given in Table 4.

Overtime option 2 for electives is not considered in combination with option 1 for emergencies. There are therefore 5 combinations of values for $\rho$ which are all combined with the 9 operational plans. We thus have a total of 45 strategies we run over a simulation horizon of $\eta=1000$ cycles of $T=28$ days. The confidence interval for most performance indicators is then less than 3 percent of the average value.

\begin{tabular}{ccccccccc}
\cline { 3 - 9 } & Option & 0 & 1 & 2 & 3 & 4 & 5 & 6 \\
\cline { 2 - 9 }$\rho_{\text {elec }}(s)$ & 1 & 1 & 1 & 1 & 1 & 1 & 1 & 1 \\
& 2 & 1.06 & 1.04 & 1.02 & 1.00 & 0.98 & 0.96 & 0.94 \\
\hline$\rho_{\text {emer }}(s)$ & 1 & 1 & 1 & 1 & 1 & 1 & 1 & 1 \\
& 2 & 1.06 & 1.06 & 1.06 & 1.06 & 1.06 & 1.06 & 1.06 \\
& 3 & 1.06 for OT, $+\infty$ for the other resources \\
\hline
\end{tabular}

Table 4. Values for parameter $\rho$ for elective and emergency patients 
Arrivals of emergency and elective patients were simulated through a Poisson process with a parameter depending on the day of the week for emergencies (Appendix B5) and a fixed weekday parameter for electives (Appendix B1). Thus, emergency patients represent about 25\% of the number of elective patients. For each scheduled patient, we drew an actual IC stay and MC stay according to the empirical distributions that were obtained from the hospital and described in Tables B3 and B4 (Appendix B).

\subsection{Numerical results}

We considered the indicators described in section 4.3 (see Table 3) and averages were computed for each option of flexible scheduling rules and each option for $\rho$ for both elective and emergency patients. Table 5 provides these averages. From the results displayed in Table 5, we may draw several comments, analyzing each strategy individually.

\begin{tabular}{|c|c|c|c|c|c|c|c|c|c|c|c|c|c|c|}
\hline & 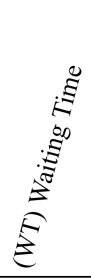 & 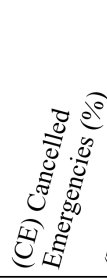 & 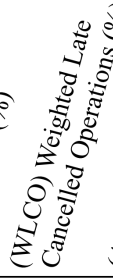 & 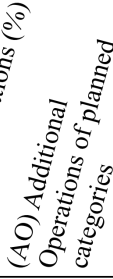 & 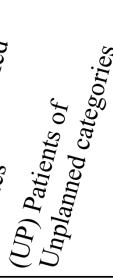 & 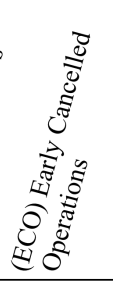 & 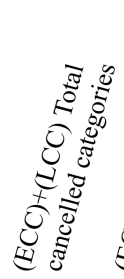 & 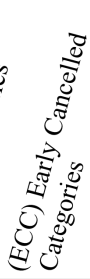 & 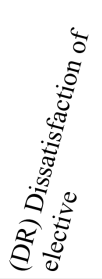 & 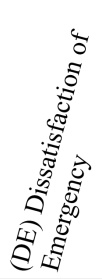 & 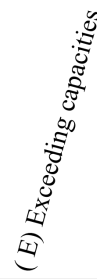 & 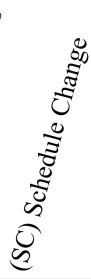 & 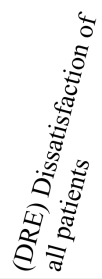 & 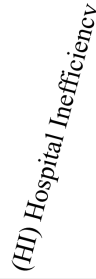 \\
\hline \multicolumn{15}{|l|}{ Overplan } \\
\hline No (O1) & 2.56 & 6.73 & 1.40 & 5.42 & 2.33 & 4.99 & 4.73 & 4.62 & 2555 & 1207 & 33.02 & 64.94 & 3762 & 6549 \\
\hline Inte & & & & & & & 1.10 & & & 1429 & & 00 & & 9533 \\
\hline Large $(\mathrm{O} 2)$ & & & & & & 11.5 & 25 & 12.0 & 21 & 160 & 16 & & 787 & 11157 \\
\hline \multicolumn{15}{|l|}{ Flexibility } \\
\hline No $(\mathrm{F} 1)$ & .80 & 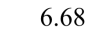 & 23 & & 0.00 & 3.28 & 5.36 & 5.25 & 6 & 197 & 32.71 & 14.12 & 840 & 3977 \\
\hline Inte & .25 & & 84 & 1 & & 9.12 & 8.48 & 8.2 & 193 & 516 & 40.59 & 80.44 & 447 & 8081 \\
\hline Large (F2) & 1.06 & 8.52 & 4.45 & 12.63 & 11.43 & 12.33 & 14.52 & 14.12 & 205 & 1527 & 42.20 & 219.21 & 585 & 15181 \\
\hline \multicolumn{15}{|l|}{$\overline{\text { Exc. Capa. El. }\left(\rho_{e l e}\right)}$} \\
\hline & 64 & & & & & & & 9. & & & & & & 72 \\
\hline Yes (option 2) & & & & & 3 & 8.37 & & 9.3. & 21 & & 78 & & & 11873 \\
\hline \multicolumn{15}{|c|}{ Exc. Capa. Em. $\left(\rho_{\text {emer }}\right)$} \\
\hline No (option 1) & 16.22 & 5.43 & & & & 8.18 & & 9.30 & 0 & 2766 & 7.75 & & 26 & 6034 \\
\hline & & & & & & & & & & & & & & 8192 \\
\hline Large (option 3) & 15.65 & 3.30 & 2.99 & 7.88 & 3.79 & 8.32 & 9.44 & 9.17 & 2204 & 591 & 62.50 & 104.79 & 2795 & 11490 \\
\hline
\end{tabular}

Table 5. Numerical results: indicators of inefficiency

Overplanning. Increasing the number of planned patients reduces the average waiting time and increases the number of cancelled emergencies as more resources are allocated to elective patients. However, late and early cancellations for elective also increase: with no overplanning, there is a stronger tendency to stick to the initial plan (see $(E C O)$ and $(E C C)$ ) or to the schedule (late cancellations represented by 2 indicators: (LCC) and (WLCO). With more planned patients, there is more freedom to allocate resources differently. Thus, overplanning tends to create more schedule instability (single indicators like (UP) and (ECO) increase with the number of planned patients). Overplanning creates more possibilities for cancelling or adding operations through the use of flexible scheduling rules. On a global basis, increasing the number of planned patients decreases the waiting time and increases the cancelled emergencies.

The improvement of waiting times compensates for the increased cancellations to a certain extent: for large overplanning the decrease of waiting time is minor compared to the one we get with intermediate overplanning therefore entailing a satisfaction decrease for elective (see index (DR)). Thus elective patients reach the best satisfaction with intermediate overplanning (however, the satisfaction of emergency patients degrades). The dissatisfaction of all patients 
reaches its minimum value for intermediate overplanning. Overplanning creates more schedule instability and -when allowed- capacities are more exceeded for larger numbers of planned patients. This results in a decrease of the overall hospital efficiency.

Flexibility. An increased flexibility decreases the overall waiting time as some patients may be replaced with others. This leads to scheduling more patients than initially planned. Additional operations $(A O)$ are only zero for the no flexibility option, and scheduled patients from unplanned categories (UP) are obviously only positive for (F2). The flexibility options induce a large increase of the schedule change indicator $(S C)$ compared to the no flexibility option. With flexibility, early and late cancellations of elective are increased and the number of cancelled emergencies also is increased. Although there are more cancellations, there are also more additional operations from other types. Therefore the average waiting time decreases, and of course, with a shorter queue length, the probability becomes much larger that there is no patient at all from a certain category, so that the session has to be cancelled.

Due to increased schedule changes, the hospital inefficiency increases as the flexibility level increases. Although there are more patient cancellations, there is also less waiting time which globally entails a lower satisfaction for emergency patients; the highest service for elective patients is reached for intermediate flexibility. Indeed large flexibility induces only a slight decrease of waiting time that does not compensate for cancellations. We obtain the best overall service for all patients with intermediate flexibility option.

Capacity excess for elective patients. This strategy uses a tight capacity when making the operational plan, but allows for capacity excess on the operation day. Therefore, we find a small increase in waiting time, but a reduction in the cancelled operations. It also reduces the cancelled emergencies because more capacities are made available for emergency. It has no significant impact on schedule changes. The hospital inefficiency is obviously increased due to the increase of capacity excess. The satisfaction of both types of patients is increased.

Capacity excess for emergencies. Increasing the capacity violations for emergency mechanically leads to a decreased number of cancelled emergencies and thus to an increased satisfaction for emergency patients. There is no significant impact on the schedule change indicators. Obviously, hospital inefficiency is increased due to capacity violations.

Table 6 provides the Pareto efficient combinations of strategies in terms of patients' dissatisfaction and hospital inefficiency. Overplanning and flexibility strategies produce Pareto efficient solutions when used in alternation: large overplanning is associated with no flexibility and no overplanning is associated with intermediate flexibility. Large flexibility never appears as an efficient solution since it produces only a slight decrease in waiting time that does not compensate for cancellations. The best alternative for patient satisfaction is large overplanning, no flexibility and large capacity excesses allowed for both types of patients. Conversely, the best hospital efficiency is reached when none of the strategies developed here is applied. Indeed, overplanning and flexibility create schedule instability that is detrimental to the hospital efficiency. 


\begin{tabular}{|c|c|c|c|c|c|}
\hline Overplan & Flexibility & $\begin{array}{l}\text { Exc. Capa. } \\
\text { Elective }\end{array}$ & $\begin{array}{l}\text { Exc. Capa. } \\
\text { Emergency }\end{array}$ & $\begin{array}{l}\text { Patients } \\
\text { Dissatisfaction } \\
(D R E)\end{array}$ & $\begin{array}{l}\text { Hospital } \\
\text { Inefficiency } \\
(H I)\end{array}$ \\
\hline Large & No & Yes & Large & 2086 & 8555 \\
\hline No & Intermediate & No & Large & 2292 & 6535 \\
\hline Large & No & No & Large & 2488 & 4931 \\
\hline Intermediate & No & No & Large & 2662 & 4753 \\
\hline No & Intermediate & No & Small & 3387 & 3344 \\
\hline Large & No & No & Small & 3483 & 1980 \\
\hline Intermediate & No & No & Small & 3507 & 1616 \\
\hline No & No & No & Small & 4288 & 1145 \\
\hline No & No & No & No & 6301 & 1088 \\
\hline
\end{tabular}

\section{Table 6. Pareto efficient strategies}

Allowing for capacity excess also decreases the hospital efficiency. There is obviously a tradeoff between patient dissatisfaction and hospital inefficiency: an increase of the hospital efficiency can only be made at the expense of a decreasing patient service. This trade-off can be better visualized in Figure 5 that plots Pareto efficient solutions as well as non-efficient solutions, where patient dissatisfaction appears along the $\mathrm{x}$-axis and hospital inefficiency, along the $\mathrm{y}$-axis. Allowing for large capacity excess especially for emergency patients provides the highest patient satisfaction and the worst hospital efficiency. With few or no capacity excess, we obtain the opposite situation.

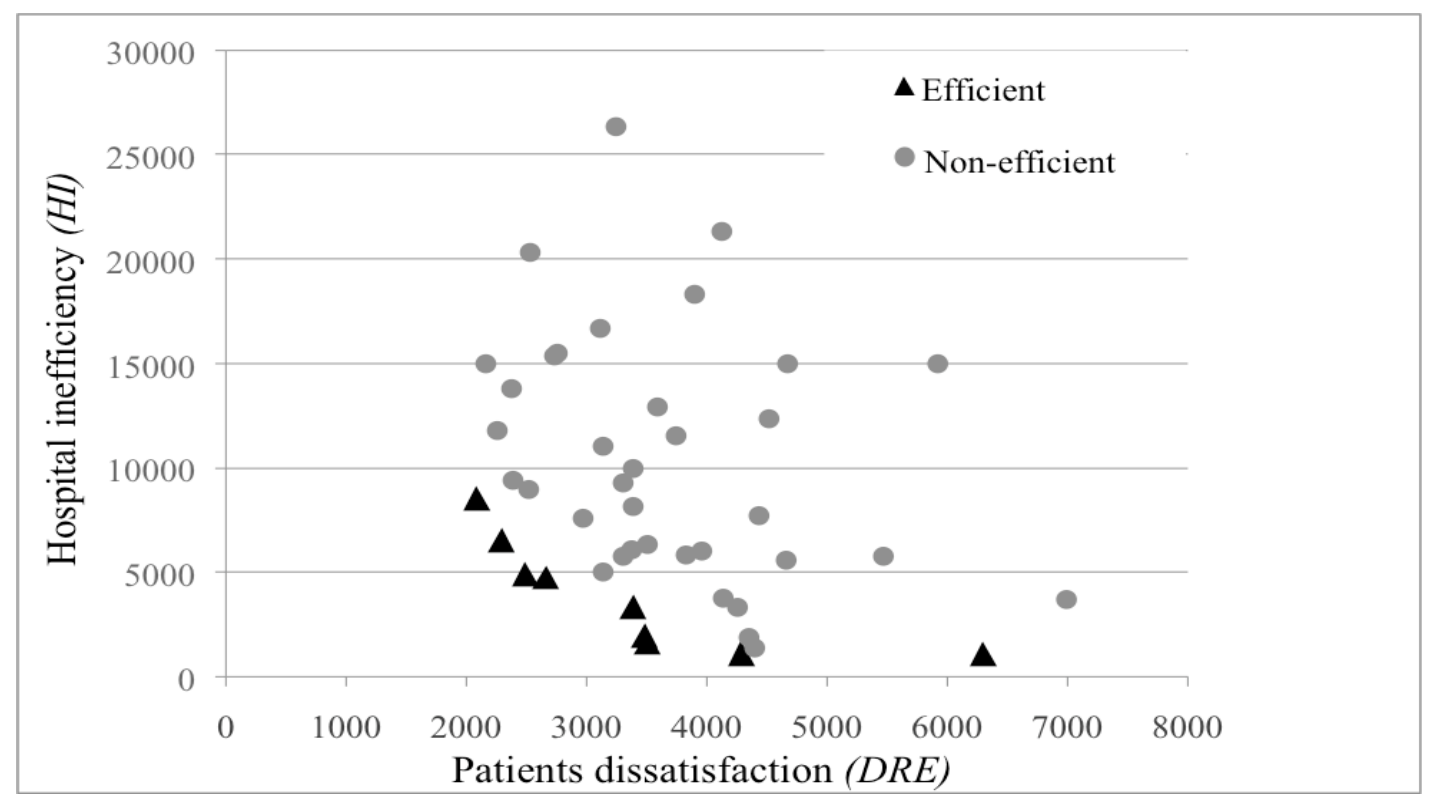

Figure 5. Efficient and non-efficient strategies 


\section{Conclusion}

In this paper, we have presented a mixed integer program to obtain a tactical plan that accounts for emergency patients by reserving capacities for these patients. Overplanning and flexibility rules were proposed to derive an operational plan from the tactical plan. To deal with daily emergency patients, we have developed a daily scheduling algorithm with cancellations rules for both elective and emergency patients. Simulation results show an obvious trade-off between patients' dissatisfaction and hospital inefficiency: increasing patient satisfaction can only be made at the expense of a decreasing hospital efficiency. Strategies behind patient satisfaction involve overplanning, intermediate flexibility (with an increased satisfaction for electives only) and soft cancellation rules (i.e. allowing for capacity excess). Such strategies lead to schedule changes and capacity excess, two components of hospital inefficiency.

Further improvements may be brought to our daily scheduling algorithm. Cancellations of elective patients are currently implemented by scanning patients category by category. However less cancellation with a better capacity allocation could be obtained by considering several combinations of elective patients as candidate for cancellations. Comparison of the proposed tactical and operational strategies with existing ones could be implemented. First, existing strategies should be adapted to our hospital context. Comparisons of strategies are indeed tedious to implement due to a lack of unifying framework that implies a high degree of customization in the design of strategies. In our paper, we have utilized Cplex to obtain a tactical plan. But most hospitals are not equipped with such a software. We could instead develop heuristics to obtain a tactical plan. This would give rise the opportunity to assess the impact of the quality of the tactical plan on the performance of our strategies.

\section{Appendix A. The daily scheduling algorithm}

We adopt the following simplified notations to describe the patient flows, resources utilization and capacities in any day $t$ (the time index is omitted in Table A1 for the sake of simplicity).

\begin{tabular}{|c|c|}
\hline$A O P$ & Already Operated Patients: set of all patients actually operated on day $t$ \\
\hline$D E A$ & $\begin{array}{l}\text { Day Emergency Arrivals: set of the estimated number of emergency patients } \\
\text { in each group, arriving on day } t \text {. The estimation is done at the beginning of the } \\
\text { day shift }\end{array}$ \\
\hline$R D E$ & $\begin{array}{l}\text { Remaining Day Emergency: set of emergency patients arriving during the } \\
\text { current day shift and waiting for an operation; }\end{array}$ \\
\hline$R N E$ & $\begin{array}{l}\text { Remaining Night Emergency: set of night emergency patients arriving during } \\
\text { the night shift and waiting for an operation; }\end{array}$ \\
\hline SEP & $\begin{array}{l}\text { Scheduled Elective Patients: set of elective patients planned for operation } \\
\text { during the current day shift; }\end{array}$ \\
\hline$I C P_{c, t}$ & Actual number of patients from category $c$ in the IC unit on day $t$ \\
\hline & Actual number of patients in MC on day $t$ \\
\hline$l_{I C}(a)$ & Deterministic length of stay in IC for a patient that has been operated \\
\hline$l_{M C}(a)$ & Deterministic length of stay in MC for a patient that has been operated \\
\hline
\end{tabular}




$\begin{array}{ll}C_{O T}^{\text {night }}, C_{O T}^{\text {day }} & \begin{array}{l}\text { Capacity of the operating theatre during the current night shift and day shift, } \\ \text { respectively (in hours); }\end{array} \\ Q_{O T}^{\text {night }}, Q_{O T}^{\text {day }} & \begin{array}{l}\text { Utilization of operating rooms during the current night shift and day shift, } \\ \text { respectively (in hours); }\end{array} \\ C_{I C}, C_{M C}, C_{N H} & \text { Other resources capacity for the current day under examination; } \\ Q_{I C}, Q_{M C}, Q_{N H} & \text { Other resources utilization in the current day; }\end{array}$

\section{Table A1. Notations}

It should be noted that sets $A O P, D E A, R D E, R N E$ and $S E P$ are of the form $\left\{a_{1}, \ldots, a_{N}\right\}$ where $a_{i}$ is the number of patients in category $i$ (to be) operated in the different sets. Dealing with emergency arrivals implies a comparison between the projected resource consumption required to operate both the emergency and elective patients and the available resources. This may lead to cancelling operations by postponing some planned elective patients and/or by sending some emergency patients to other hospitals. In the latter case, we assume that only emergency patients arriving during the day may be sent to another hospital whereas night emergency arrivals are all admitted for operation.

Each time a patient is operated, we provide an estimation of his length of stay in IC $\left(l_{I C}\right)$ and in $\mathrm{MC}\left(l_{M C}\right)$. We consider these lengths of stay as deterministic. For patients in the set $A O P$ that have been operated, the consumption of IC and MC resources is no longer based on probabilities. Let $I C P_{c, t}^{t-j}$ be the number of patients from category $c$, operated on day $t-j$ that are (still) in the IC unit on day $t$ :

$$
I C P_{c, t}^{t-j}=\operatorname{Card}\left\{a \in A O P_{c, t-j} \mid l_{I C}(a) \geq j+1\right\}, \quad t=1, \ldots, T, \quad j=0, \ldots, L_{I C}^{\max }-1
$$

The number of occupied beds in IC on day $t$ is: $I C P_{t}=\sum_{c=1}^{N} \sum_{j=0}^{L_{I C}^{\max }-1} I C P_{c, t}^{t-j}$.

For the patients in $\mathrm{MC}$, we do not need any information on the categories; the number of patients in $\mathrm{MC}$ on day $t$ is

$$
M C P_{t}=\sum_{j=0}^{L_{M C}^{\max }-1} \operatorname{Card}\left\{a \in A O P_{t-j} \mid l_{I C}(a)+l_{M C}(a) \geq j+1 ; l_{I C}(a) \leq j\right\} \quad t=1, \ldots, T
$$

Here we do not take into account the preoperative stay of patients that will be operated on day $t+l_{c}$ as we do not know yet the exact number of these patients due to the postponement rule that we apply in the daily scheduling algorithm. Below the algorithm is described step by step. 
- Start with an empty system, i.e. set $A O P=S E P=R D E=R N E=\varnothing$ and $Q_{O T}^{\text {night }}=0$

- Arriving patients are put in the waiting list and removed when scheduled

- Schedule the operation of elective patients for days $t=1, \ldots, 7$ according to the method described in section 4.1. This provides the sets $S E P_{1}, \ldots, S E P_{7}$ of scheduled elective patients.

\section{Night shift $t$ : dealing with night emergencies}

Step 1. At the beginning of the night shift, no patient has been operated yet on day $t$, thus we set $a_{c, t}^{A O P}=0, \quad c=1 . N$. Fill $R N E$ with all night emergency arrivals and go to Step 2.

Step 2. If $R N E \neq \varnothing$ then define $\underline{c}$ the category of the earliest arrived night emergency patient in the set $R N E$ and go to Step 3. Else go to Step 4.

Step 3. Operate the earliest arrived patient (category $\underline{c}$ ) and actualize:

-Set $Q_{O T}^{\text {night }}=Q_{O T}^{\text {night }}+S_{\underline{c}}$

-Update $R N E=\left\{a_{1}^{R N E}, \ldots, a_{\underline{c}}^{R N E}-1, \ldots, a_{N}^{R N E}\right\}$

-Update $A O P_{t}=\left\{a_{1, t}^{A O P}, \ldots, a_{\underline{c}, t}^{A O P}+1, \ldots, a_{N, t}^{A O P}\right\}$ (set of operated patients on day $t$ )

-Estimate a length of stay in IC and MC for this patient

- Go to Step 2

Step 4. End of the night shift

-If $Q_{O T}^{\text {night }}>C_{O T}^{\text {night }}$ this means that a night patient undergoes an operation that has started during the night shift and that will be ending during the day shift therefore consuming OT resources from the day shift. Thus, the initial value of $Q_{O T}^{\text {day }}$ equals $Q_{O T}^{\text {night }}-C_{O T}^{\text {night }}$. All night emergencies are taken in charge and we allow for possible overuse of other resources than OT in subsequent days. Thus the set $R N E$ of remaining night emergencies is always empty at the end of any night shift.

- Go to Step 5

\section{Day shift $t$ : operation decisions about elective patients}

Step 5. In this step, we compute the projected use of each resource over a horizon of $S$ periods by (i) the already operated patients, (ii) the remaining night emergencies and (iii) the estimated day emergency arrivals (unknown at the beginning of the day shift so evaluated).

The resource use entailed by the operation of an additional elective patient will be evaluated in the next step.

-Set $Q_{O T}^{d a y}=\max \left(0, Q_{O T}^{\text {night }}-C_{O T}^{\text {night }}\right)$ 
-Estimate the number of day emergency arrivals in each category (set $\left.D E A=\left\{a_{1, t}^{D E A}, \ldots, a_{N, t}^{D E A}\right\}\right)$

-The projected OT use is given by

$$
Q_{O T, t}=Q_{O T}^{d a y}+\sum_{c=1}^{N} s_{c} a_{c, t}^{D E A} ;
$$

-The projected uses of other resources on day $t+s$, with $s=0, \ldots, S$ are written as

$$
\begin{aligned}
& Q_{I C, t+s}=I C P_{t+s}+\sum_{c=1}^{N} p_{I C, c, s} \cdot a_{c, t}^{D E A}+\sum_{c=1}^{N} \sum_{j=0}^{s-1} p_{I C, c, j} \cdot a_{c, t+s-j}^{S E P} ; \\
& Q_{M C, t+s}=M C P_{t+s}+\sum_{c=1}^{N} p_{M C, c, s} \cdot a_{c, t}^{D E A}+\sum_{c=1}^{N} \sum_{j=0}^{s-1} p_{M C, c, j} \cdot a_{c, t+s-j}^{S E P}+\sum_{c=1}^{N} \sum_{j=1}^{l_{c}} a_{c, t+s+j}^{S E P} ; \\
& Q_{N H, t+s}=\sum_{c=1}^{N} \sum_{j=0}^{L_{I C}^{\max }-1} w_{c, j} \cdot I C P_{c, t+s}^{t+s-j}+\sum_{c=1}^{N} w_{c, s} \cdot p_{I C, c, s} \cdot a_{c, t}^{D E A}+\sum_{c=1}^{N} \sum_{j=0}^{s-1} w_{c, j} \cdot p_{I C, c, j} \cdot a_{c, t+s-j}^{S E P},
\end{aligned}
$$

where $I C P_{c, t}^{t-j}$ is defined in (10) and $M C P_{t}$, in (11)

- Set the Remaining Day Emergencies to zero: $R D E=\{0, \ldots, 0\}$

-Set the Remaining Elective Patients (set $R E P$ ) equal to the set of Scheduled Elective Patients: $R E P=S E P_{t}$

- Go to Step 6

Step 6. Consider all the patients from the set $S E P_{t}$ one by one and category by category, starting with the least crowded one and ending with the most crowded category. Let $\underline{c}$ be the current category under examination.

If there is no more patient to be examined from the set $S E P_{t}$ then go to Step 9. Else: compute the projected use of each resource on day $t$, entailed by the operation of an additional elective patient.

- Let $a \in R E P$ be a remaining elective patient of category $\underline{c}$ picked for possible operation on day $t$.

-Estimate his lengths of stay $l_{I C}(a)$ and $l_{M C}(a)$ in both IC and MC units

-Actualize the OT use on day $t$

$$
Q_{O T, t}=Q_{O T, t}+s_{\underline{c}}
$$

-Actualize the other resources use for $s=0, \ldots, S$

$$
\begin{aligned}
& Q_{I C, t+s}=Q_{I C, t+s}+1 \text { if } l_{I C}(a) \geq s+1, \text { else } Q_{I C, t+s}=Q_{I C, t+s} \\
& Q_{M C, t+s}=Q_{M C, t+s}+1 \text { if } l_{I C}(a)+l_{M C}(a) \geq s+1 \text { and } l_{I C}(a) \leq s, \text { else } Q_{M C, t+s}=Q_{M C, t+s} \\
& Q_{N H, t+s}=Q_{N H, t+s}+w_{\underline{c}, t+s} \text { if } l_{I C}(a) \geq s+1, \text { else } Q_{N H, t+s}=Q_{N H, t+s}
\end{aligned}
$$

- Go to Step 7. 
Step 7. If $Q_{I C, t+s} \leq \rho(s) \cdot C_{I C, t+s}$ and $Q_{M C, t+s} \leq \rho(s) \cdot C_{M C, t+s}$ and $Q_{N H, t+s} \leq \rho(s) \cdot C_{N H, t+s}$ and $Q_{O T, t} \leq \rho(0) \cdot C_{O T, t}$ then the capacity is not exceeded so operate patient $a \in R E P$

- Set $A O P_{t}=A O P_{t} \cup\{a\}$

- Set $R E P=R E P \backslash\{a\}$

-Go to Step 6

Else go to Step 8 .

Step 8. At least one of the capacity is violated, thus patient $a \in R E P$ is added to the waiting list of patients to be scheduled 7 days later (it is possible that some patients of the waiting list on day $t+7$ are not operated)

- Update the resources use by removing the consumption entailed by patient $a \in R E P$ :

$$
Q_{O T, t}=Q_{O T, t}-S_{\underline{c}}
$$

For IC, MC and $\mathrm{NH}$ and for $s=0, \ldots, S$ we have

$$
\begin{aligned}
& Q_{I C, t+s}=Q_{I C, t+s}-1 \text { if } l_{I C}(a) \geq s+1, \text { else } Q_{I C, t+s}=Q_{I C, t+s} \\
& Q_{M C, t+s}=Q_{M C, t+s}-1 \text { if } l_{I C}(a)+l_{M C}(a) \geq s+1 \text { and } l_{I C}(a) \leq s, \text { else } Q_{M C, t+s}=Q_{M C, t+s} \\
& Q_{N H, t+s}=Q_{N H, t+s}-w_{\underline{c}, t+s} \text { if } l_{I C}(a) \geq s+1, \text { else } Q_{N H, t+s}=Q_{N H, t+s}
\end{aligned}
$$

-Go to Step 6

\section{Day shift $t$ : operation decisions about emergency patients}

Step 9. Now the decisions about the operation of elective patients have been taken based upon projected resources use by estimated emergency arrivals, we decide upon emergency operations on the basis of actual emergency arrivals. We thus remove from the projected resources use, the estimated consumption by emergency patients:

-The projected OT use is given by

$$
Q_{O T, t}=Q_{O T, t}-\sum_{c=1}^{N} s_{c} a_{c, t}^{D E A} ;
$$

-The projected uses of other resources on day $t+s$, with $s=0, \ldots, S$ are written as

$$
\begin{aligned}
& Q_{I C, t+s}=Q_{I C, t+s}-\sum_{c=1}^{N} p_{I C, c, s} \cdot a_{c, t}^{D E A} ; \\
& Q_{M C, t+s}=Q_{M C, t+s}-\sum_{c=1}^{N} p_{M C, c, s} \cdot a_{c, t}^{D E A} ; \\
& Q_{N H, t+s}=Q_{N H, t+s}-\sum_{c=1}^{N} w_{c, s} \cdot p_{I C, c, s} \cdot a_{c, t}^{D E A},
\end{aligned}
$$


-Fill $R D E$ with all day emergency arrivals and go to Step 10.

Step 10. Consider every patient of $R D E$ in his arrival order (the first patient arriving at the emergency department should also be the first patient operated). If all patients of $R D E$ have been examined then go to Step 14. Else, define $\underline{c}$ as the category of the current patient under examination and go to Step 11.

Step 11. Compute the resources use entailed by an additional patient:

- Let $a \in R D E$ be a remaining day emergency of category $\underline{c}$ picked for possible operation on day $t$.

-Estimate his lengths of stay $l_{I C}(a)$ and $l_{M C}(a)$ in both IC and MC units

-Actualize the OT use on day $t$ (identical to Step 6)

$$
Q_{O T, t}=Q_{O T, t}+s_{\underline{c}}
$$

-Actualize the other resources use for $s=0, \ldots, S$ (identical to Step 6)

$$
Q_{I C, t+s}=Q_{I C, t+s}+1 \text { if } l_{I C}(a) \geq s+1 \text {, else } Q_{I C, t+s}=Q_{I C, t+s}
$$$$
Q_{M C, t+s}=Q_{M C, t+s}+1 \text { if } l_{I C}(a)+l_{M C}(a) \geq s+1 \text { and } l_{I C}(a) \leq s \text {, else } Q_{M C, t+s}=Q_{M C, t+s}
$$$$
Q_{N H, t+s}=Q_{N H, t+s}+w_{\underline{c}, t+s} \text { if } l_{I C}(a) \geq s+1 \text {, else } Q_{N H, t+s}=Q_{N H, t+s}
$$

- Go to Step 12.

Step 12. If $Q_{I C, t+s} \leq \rho \cdot C_{I C, t+s}$ and $Q_{M C, t+s} \leq \rho \cdot C_{M C, t+s}$ and $Q_{N H, t+s} \leq \rho \cdot C_{N H, t+s}$ and $Q_{O T, t} \leq \rho \cdot C_{O T, t}$ then the capacity is not exceeded so operate patient $a \in R D E$. Compared to Step 7, it should be noted that here, coefficient $\rho$ is constant overtime. Coefficient $\rho$ is related to the probability of postpone/cancel a patient that is scheduled. No emergency patient can be cancelled during future days as only in the current day we have emergency patients.

- Set $A O P_{t}=A O P_{t} \cup\{a\}$

- Set $R D E=R D E \backslash\{a\}$

- Go to Step 10

Else go to Step 13.

Step 13. At least one of the capacity is violated, thus all patients of the set $R D E$ (Remaining Day Emergency) are sent to other hospitals and the patient $a \in R D E$ selected in Step 11 is not operated so actualize the use of resources: At least one of the capacity is violated, thus patient $a \in R E P$ is added to the waiting list of patients to be scheduled 7 days later (it is possible that some of the patients of the waiting list on day $t+7$ are not operated)

-Update the resources use by removing the consumption entailed by patient $a \in R D E$ :

$$
Q_{O T, t}=Q_{O T, t}-s_{\underline{c}}
$$


For IC, MC and $\mathrm{NH}$ and for $s=0, \ldots, S$ we have

$$
\begin{aligned}
& Q_{I C, t+s}=Q_{I C, t+s}-1 \text { if } l_{I C}(a) \geq s+1, \text { else } Q_{I C, t+s}=Q_{I C, t+s} \\
& Q_{M C, t+s}=Q_{M C, t+s}-1 \text { if } l_{I C}(a)+l_{M C}(a) \geq s+1 \text { and } l_{I C}(a) \leq s, \text { else } Q_{M C, t+s}=Q_{M C, t+s} \\
& Q_{N H, t+s}=Q_{N H, t+s}-w_{\underline{c}, t+s} \text { if } l_{I C}(a) \geq s+1, \text { else } Q_{N H, t+s}=Q_{N H, t+s}
\end{aligned}
$$

- Go to Step 10

Step 14.

-All patients in the set $R D E$ are sent to other hospitals.

- At the end of the day shift, all resources use corresponds to actual use and is no longer subject to modification except for MC due to preoperative days. Indeed, in our case with only one preoperative day, this means that in the morning, after the possible postponement of electives today, and before the admission of emergencies during the day, we hypothetically admit the electives for tomorrow and include their $\mathrm{MC}$ consumption in this preoperative day. If their operation is cancelled on the next day, this pre-operative day has been a waste.

- Set $Q_{O T}^{\text {night }}=\max \left(0, Q_{O T}^{d a y}-C_{O T}^{d a y}\right)$ : the operation of some day emergency patients may continue during the night shift therefore consuming night resources.

- According to the method described in section 4.1, define the planned elective patients to be scheduled on day $t+S+1$ by considering both the actual arriving patients and the waiting list of day $t+S+1$

- Set $t=t+1$

- Go to Step 1 if $t \leq T$, else Stop.

\section{Appendix B. The data}

The data are based on the Thorax Center Rotterdam, also partly used in Vissers et al. (2005) and Adan et al (2009). Recent data shows some shifts between categories, but confirms the major characteristics. Patients have been grouped in $N=8$ categories, each of these being pretty homogeneous in terms of consumption of OT and IC resources. Table B1 provides for each patient category, examples of the surgical procedures performed and the expected number of hours to operate one patient of each group $\left(s_{c}\right)$. In the penultimate column, we give the number of pre-operative days for each category $\left(l_{c}\right)$. The last column displays the average number of patients for each category.

\begin{tabular}{llccc}
\hline \multicolumn{1}{c}{ Patient group $(\boldsymbol{c}), \boldsymbol{N}=\boldsymbol{8}$} & \multicolumn{1}{c}{ Example procedures } & $\begin{array}{c}\text { OT hours } \\
\left(s_{c}\right)\end{array}$ & $\begin{array}{c}\text { Pre-op. day } \\
\left(l_{c}\right)\end{array}$ & $\begin{array}{c}\text { Average \# } \\
\text { patients }\end{array}$ \\
\hline (1) Child simple & Closure ventricular septal defect & 4 & 0 & 7.36 \\
(2) Child complex & Arterial switch & 8 & 0 & 9.36 \\
(3) Adult, short OT, short IC & Coronary bypass (CABG) & 4 & 1 & 66.00 \\
(4) Adult, long OT, short IC & Mitral valve plasty & 8 & 1 & 12.73
\end{tabular}


(5) Adult, short OT, middle IC CABG with expected medium IC $\quad 4 \quad 2.64$

(6) Adult, long OT, middle IC Heart transplant $\quad 8 \quad 1 \quad 1.55$

(7) Adult, long OT, long IC $\quad$ Thoraco-abdominal aneurysm $\quad 8 \quad 1 \quad 0.36$

$\begin{array}{llll}\text { (8) Adult, very short OT, no IC Cervical mediastinoscopy } & 2 & 1 & 6.91\end{array}$

Table B1. Patient groups, OT hours, pre-operative days and 4-week volume

\section{Available resources}

For each of the resources, there exists a maximum available capacity per day $\left\{C_{r, t}\right\}$ which is displayed in Table B2. The data apply to every week in the 4-week planning cycle $(T=28)$.

\begin{tabular}{lcccc}
\cline { 2 - 5 } Day & OT hours & $\begin{array}{c}\text { Number of } \\
\text { IC beds }\end{array}$ & $\begin{array}{c}\text { Number of } \\
\text { MC beds }\end{array}$ & $\begin{array}{c}\text { IC Nursing } \\
\text { hours }\end{array}$ \\
\hline Monday & 36 & 10 & 36 & 133 \\
Tuesday & 36 & 10 & 36 & 133 \\
Wednesday & 36 & 10 & 36 & 133 \\
Thursday & 36 & 10 & 36 & 133 \\
Friday & 36 & 10 & 36 & 133 \\
Saturday & 8 & 5 & 36 & 65 \\
Sunday & 8 & 5 & 36 & 65 \\
\hline
\end{tabular}

Table B2. Maximum capacity for each resource in each day

Four operating theatres are available nine hours per day during the week days. On week-ends, only one operating theatre is available to operate emergency patients. The IC unit has 10 beds available throughout the working week and 5 beds during the weekend. The MC unit has 36 beds available every day. The available IC nursing staff is matched with the number of IC beds.

The IC nursing hours $\left(w_{c, t}\right)$ required per day for patients of categories 1 to 7 are estimated to 12 hours throughout their stay in the IC unit unless for the second day in IC and for categories 5, 6 and 7 for which the needs are 24 hours. For category 7 there is also a need of 24 hours on day 3 . Patients from category 8 only require 3 hours of IC nursing per day whatever their length of stay in IC. The longest length of stay in IC equals 10 days $\left(L_{I C}^{\max }=9\right)$.

\section{Relative weights in the objective function}

The importance of each resource has been assessed by the stakeholders in the hospital who decided to allocate to each of them a certain absolute weight denoted by $g_{r}$, with $g_{O T}=8, g_{I C}=10, g_{M C}=3, g_{N H}=5$. The relative weight $\alpha_{r}$ for resource $r$ is defined as

$$
\alpha_{r}=\frac{\frac{g_{r}}{\sum_{j=1, \ldots, T} C_{r, j}}}{\sum_{r=\{O T, I C, M C, N H\}} \frac{g_{r}}{\sum_{j=1, \ldots, T} C_{r, j}}}
$$


where $C_{r, j}$ is the maximum capacity for resource $r$ in period $j$. For the OT resource, an exception is made as we do no include the week ends in the computation because elective patients can only be operated during the week days.

\section{Length of stay}

We use a stochastic length of stay for IC and MC, based on empirical data of the year 2006 including a sample of 576 patients. Table B3 provides information on the length of stay distribution at the IC unit for each patient group $\left(p_{I C, c, t}\right)$. For instance, any patient of category 8 has $21 \%$ chance to be at the IC unit zero day after operation, that is the day of operation $\left(p_{I C, 8,0}=0.21\right)$. In such a case, patients will stay overnight at the IC.

\begin{tabular}{lrrrrrrrrrr}
\hline Day & 0 & 1 & 2 & 3 & 4 & 5 & 6 & 7 & 8 & 9 \\
\hline (1) Child simple & 0.93 & 0.07 & 0.05 & 0.02 & 0 & 0 & 0 & 0 & 0 & 0 \\
(2) Child complex & 1 & 0.09 & 0.02 & 0 & 0 & 0 & 0 & 0 & 0 & 0 \\
(3) Adult, short OT, short IC & 0.99 & 0.16 & 0.05 & 0.02 & 0.01 & 0.01 & 0.01 & 0 & 0 & 0 \\
(4) Adult, long OT, short IC & 1 & 0.19 & 0.09 & 0.04 & 0.04 & 0.03 & 0.01 & 0.01 & 0.01 & 0.01 \\
(5) Adult, short OT, middle IC & 1 & 0.2 & 0.13 & 0.07 & 0.07 & 0.07 & 0.07 & 0 & 0 & 0 \\
(6) Adult, long OT, middle IC & 1 & 1 & 0.86 & 0.43 & 0.29 & 0.14 & 0.14 & 0.14 & 0 & 0 \\
(7) Adult, long OT, long IC & 1 & 1 & 1 & 1 & 1 & 1 & 1 & 0 & 0 & 0 \\
(8) Adult, very short OT, no IC & 0.21 & 0 & 0 & 0 & 0 & 0 & 0 & 0 & 0 & 0 \\
\hline
\end{tabular}

\section{Table B3. Length of stay distribution at IC per patient group}

Table B4 displays the same information type for the $\mathrm{MC}\left(p_{M C, c, t}\right)$. For instance, $p_{M C, 6,23}=0.29$ means that any patient from category 6 has $29 \%$ chance to be at the MC unit 23 days after the operation. We have $L_{M C}^{\max }=27$ days.

\begin{tabular}{|c|c|c|c|c|c|c|c|c|c|c|c|c|c|c|}
\hline Day & 0 & 1 & 2 & 3 & 4 & 5 & 6 & 7 & 8 & 9 & 10 & 11 & 12 & 13 \\
\hline (1) Child simple & 0.05 & 0.26 & 0.26 & 0.26 & 0.26 & 0.21 & 0.14 & 0.07 & 0 & 0 & 0 & 0 & 0 & 0 \\
\hline (2) Child complex & 0 & 0.13 & 0.17 & 0.17 & 0.17 & 0.17 & 0.17 & 0.13 & 0.09 & 0.08 & 0.06 & 0.06 & 0.06 & 0.06 \\
\hline (3) Adult, short OT, short IC & 0.01 & 0.84 & 0.94 & 0.97 & 0.95 & 0.66 & 0.42 & 0.29 & 0.21 & 0.15 & 0.11 & 0.09 & 0.07 & 0.05 \\
\hline (4) Adult, long OT, short IC & 0 & 0.78 & 0.88 & 0.93 & 0.93 & 0.84 & 0.68 & 0.53 & 0.35 & 0.25 & 0.22 & 0.19 & 0.18 & 0.13 \\
\hline (5) Adult, short OT, middle IC & 0 & 0.8 & 0.87 & 0.93 & 0.93 & 0.87 & 0.8 & 0.6 & 0.6 & 0.4 & 0.33 & 0.33 & 0.13 & 0.07 \\
\hline (6) Adult, long OT, middle IC & 0 & 0 & 0.14 & 0.57 & 0.71 & 0.86 & 0.86 & 0.86 & 1 & 1 & 0.86 & 0.86 & 0.86 & 0.86 \\
\hline (7) Adult, long OT, long IC & 0 & 0 & 0 & 0 & 0 & 0 & 0 & 1 & 1 & 1 & 1 & 1 & 1 & 1 \\
\hline (8) Adult, very short OT, no IC & 0.64 & 0.54 & 0.41 & 0.26 & 0.13 & 0.1 & 0.08 & 0.08 & 0.03 & 0.03 & 0 & 0 & 0 & 0 \\
\hline Day & 14 & 15 & 16 & 17 & 18 & 19 & 20 & 21 & 22 & 23 & 24 & 25 & 26 & 27 \\
\hline (1) Child simple & 0 & 0 & 0 & 0 & 0 & 0 & 0 & 0 & 0 & 0 & 0 & 0 & 0 & 0 \\
\hline (2) Child complex & 0.06 & 0.04 & 0.02 & 0.02 & 0.02 & 0 & 0 & 0 & 0 & 0 & 0 & 0 & 0 & 0 \\
\hline (3) Adult, short OT, short IC & 0.04 & 0.02 & 0.02 & 0.01 & 0.01 & 0.01 & 0.01 & 0 & 0 & 0 & 0 & 0 & 0 & 0 \\
\hline (4) Adult, long OT, short IC & 0.1 & 0.06 & 0.06 & 0.06 & 0.04 & 0.04 & 0.04 & 0.03 & 0.03 & 0.01 & 0.01 & 0.01 & 0.01 & 0.01 \\
\hline (5) Adult, short OT, middle IC & 0.07 & 0.07 & 0 & 0 & 0 & 0 & 0 & 0 & 0 & 0 & 0 & 0 & 0 & 0 \\
\hline (6) Adult, long OT, middle IC & 0.71 & 0.71 & 0.71 & 0.71 & 0.57 & 0.57 & 0.57 & 0.43 & 0.43 & 0.29 & 0.14 & 0 & 0 & 0 \\
\hline (7) Adult, long OT, long IC & 1 & 1 & 1 & 0 & 0 & 0 & 0 & 0 & 0 & 0 & 0 & 0 & 0 & 0 \\
\hline (8) Adult, very short OT, no IC & 0 & 0 & 0 & 0 & 0 & 0 & 0 & 0 & 0 & 0 & 0 & 0 & 0 & 0 \\
\hline
\end{tabular}

\section{Table B4. Length of stay distribution at MC per patient group}




\section{Emergency arrival}

Emergency patients arrive at a certain rate $\beta_{c, t}$ and can also be classified in the same categories as elective patients. Table B5 gives the arrival rates for each category and each day of the week. The data apply to every week in the 4-week planning cycle. The probability $q_{c, t}$ that an emergency patient of category $c$ arrives during the day shift $t$ is set to 0.8 , for all categories and all days $\left(q_{c, t}=0.8, \forall c=1 . .8, \forall t=1 . .28\right)$.

\begin{tabular}{lrrrrrrr}
\hline Day & 1 & 2 & 3 & 4 & 5 & 6 & 7 \\
\hline (1) Child simple & 0.1 & 0.1 & 0.05 & 0.05 & 0.08 & 0.06 & 0.12 \\
(2) Child complex & 0.1 & 0.12 & 0.15 & 0.05 & 0.08 & 0.16 & 0.12 \\
(3) Adult, short OT, short IC & 0.5 & 0.7 & 0.45 & 0.44 & 0.5 & 0.3 & 0.4 \\
(4) Adult, long OT, short IC & 0.1 & 0.12 & 0.11 & 0.09 & 0.08 & 0.1 & 0.12 \\
(5) Adult, short OT, middle IC & 0.01 & 0.02 & 0.04 & 0.02 & 0.02 & 0.01 & 0.01 \\
(6) Adult, long OT, middle IC & 0.01 & 0.02 & 0.03 & 0.02 & 0.02 & 0.01 & 0.01 \\
(7) Adult, long OT, long IC & 0 & 0 & 0.01 & 0 & 0 & 0 & 0 \\
(8) Adult, very short OT, no IC & 0.1 & 0.05 & 0.05 & 0.05 & 0.04 & 0.06 & 0.1 \\
\hline
\end{tabular}

Table B5. Emergency arrival rates

\section{Number of patients}

We consider 3 overplanning options: O1, O2 and O3. Option O1 (no overplanning) simply consists in rounding up the average number of arriving patients during a 4-week cycle. Table B6 exhibits for each category the number $\left(P_{c}\right)$ of patients to be planned in each option.

\begin{tabular}{lccc}
\hline Overplanning option & $\mathrm{O} 1$ & $\mathrm{O} 2$ & $\mathrm{O} 3$ \\
\hline (1) Child simple & 8 & 9 & 9 \\
(2) Child complex & 10 & 11 & 11 \\
(3) Adult, short OT, short IC & 67 & 70 & 68 \\
(4) Adult, long OT, short IC & 13 & 15 & 14 \\
(5) Adult, short OT, middle IC & 3 & 4 & 4 \\
(6) Adult, long OT, middle IC & 2 & 3 & 3 \\
(7) Adult, long OT, long IC & 1 & 2 & 1 \\
(8) Adult, very short OT, no IC & 7 & 9 & 8 \\
\hline
\end{tabular}

Table B6. Number of patients to be planned

\section{Target utilization of resources}

Defining a target level of utilization for each resource lower than $100 \%$ of the capacity allows for dealing with emergencies and fluctuations in number of patients. In our experiment, we consider three overplanning options, which leads to three situations of demand for both elective and emergency care. We therefore chose 3 sets of reasonable values for the targets $\left(R_{r, t}\right)$ as exhibited in Table B7. 


\begin{tabular}{llrrrrrrr}
\hline Overplan. Option & Day & 1 & 2 & 3 & 4 & 5 & 6 & 7 \\
\hline \multirow{3}{*}{ O1 (no over) } & OT hours & 30.19 & 30.19 & 30.19 & 30.19 & 30.19 & 3.01 & 3.46 \\
& IC beds & 7.22 & 7.22 & 7.22 & 7.22 & 7.22 & 3.61 & 3.61 \\
& MC beds & 29.13 & 29.13 & 29.13 & 29.13 & 29.13 & 29.13 & 29.13 \\
& IC Nursing hours & 88.92 & 88.92 & 88.92 & 88.92 & 88.92 & 43.46 & 43.46 \\
\hline \multirow{3}{*}{ O2 (over) } & OT hours & 33.39 & 33.39 & 33.39 & 33.39 & 33.39 & 3.01 & 3.46 \\
& IC beds & 8.13 & 8.13 & 8.13 & 8.13 & 8.13 & 4.06 & 4.06 \\
& MC beds & 32.1 & 32.1 & 32.1 & 32.1 & 32.1 & 32.1 & 32.1 \\
& IC Nursing hours & 101.32 & 101.32 & 101.32 & 101.32 & 101.32 & 49.51 & 49.51 \\
\hline \multirow{3}{*}{ O3 (interm.) } & OT hours & 32.09 & 32.09 & 32.09 & 32.09 & 32.09 & 3.01 & 3.46 \\
& IC beds & 7.66 & 7.66 & 7.66 & 7.66 & 7.66 & 3.83 & 3.83 \\
& MC beds & 30.8 & 30.8 & 30.8 & 30.8 & 30.8 & 30.8 & 30.8 \\
& IC Nursing hours & 94.8 & 94.8 & 94.8 & 94.8 & 94.8 & 46.33 & 46.33 \\
\hline
\end{tabular}

B7. Target levels of resource utilization for each demand instance

Again, the data apply to every week in the 4-week planning cycle. For weekdays, the target is the expected resource use under the assumption that all planned slots are used. For the IC beds and IC-nursing, there is a 50 percent correction for the weekend. For the OT, the weekend target is based on the expected emergency patients only.

\section{References}

Adan, I., Vissers, J., 2002. Patient mix optimisation in hospital admission planning: a case study. International Journal of Operations and Production Management, 22, 445-461.

Adan, I, J. Bekkers, N. Dellaert, J. Vissers and X. Yu, 2009. Patient mix optimisation and stochastic resource requirements: A case study in cardiothoracic surgery planning. Health Care Management Science, 12, 129-141.

Belien, J., Demeulemeester, E., 2007. Building cyclic master surgery schedules with levelled resulting bed occupancy. European Journal of Operational Research, 176, 1185-1204.

Cochran, J.K., Roche, K.T., 2009. A multi-class queuing network analysis methodology for improving hospital emergency department performance. Computers and Operations Research, 36(5), 1497-1512.

Harper, P.R., (2002). A framework for operational modelling of hospital resources. Health Care Management Science, 5, 165-173.

Lamiri, M., X. Xie, A. Dolgui and F. Grimaud, 2008. A stochastic model for operating room planning with elective and emergency demand for surgery, European Journal of Operational Research, 185(3), 1026-1037.

Litvak, N., M. van Rijsbergen, R.J. Boucherie and M. Vanhoudenhoven, 2008. Managing the overflow of intensive care patients, European Journal of Operational Research, 185(3), 9981010. 
Oostrum, J.M van, M. Vanhoudenhoven, J.L. Hurink, E.W. Hans, G. Wullink and G. Kazemier. A master surgery scheduling approach for cyclic scheduling in operating room departments. $O R$ Spectrum, 2008.

Ridge, J., Jones, S., Nielsen, M., Shahani, A., 1998. Capacity planning for intensive care unit. European Journal of Operational Research, 105, 346-355.

Utley, M., Gallivan, S., Treasure, T., Valencia, O, 2003. Analytical methods for calculating the capacity required to operate an effective booked admissions policy for elective inpatient services. Health Care Management Science, 6, 97-104.

Vissers, J., Adan, I., Bekkers, J., 2005. Patient mix optimization in cardiothoracic surgery planning: a case study. IMA Journal of Management Mathematics, 16, 281-304.

Vissers, J., Adan, I., Dellaert, N., 2007. Developing a platform for comparison of hospital admission systems: An Illustration. European Journal of Operational Research, 180, 1290-1301. 